\title{
Dissipation in small systems: Landau-Zener approach
}

\author{
Felipe Barra ${ }^{1}$ and Massimiliano Esposito ${ }^{2}$ \\ ${ }^{1}$ Departamento de Fisica, Facultad de Ciencias Fisicas y Mathematicas, Universidad de Chile, 837.0415 Santiago, Chile \\ ${ }^{2}$ Complex Systems and Statistical Mechanics, Physics and Materials Science, University of Luxembourg, L-1511 Luxembourg
}

(Received 4 February 2016; published 13 June 2016)

\begin{abstract}
We establish a stochastic thermodynamics for a Fermionic level driven by a time-dependent force and interacting with initially thermalized levels playing the role of a reservoir. The driving induces consecutive avoided crossings between system and reservoir levels described within Landau-Zener theory. We derive the resulting system dynamics and thermodynamics and identify energy, work, heat, entropy, and dissipation. Our theory perfectly reproduces the numerically exact quantum work statistics obtained using a two point measurements approach of the total energy and provides an explicit expression for the dissipation in terms of diabatic transitions.
\end{abstract}

DOI: 10.1103/PhysRevE.93.062118

\section{INTRODUCTION}

The study of quantum mechanical (QM) diabatic transitions, also called Landau-Zener (LZ) transitions, played a major role in many areas of quantum physics since the seminal work of Refs. [1-4] (see, e.g., the introduction of Ref. [5]). They occur between the time-dependent eigenstates of quantum systems driven by time-dependent forces and are often interpreted as a signature of dissipative processes [6-9]. In this paper, we investigate their connection to dissipation within the framework of quantum fluctuation relations such as the quantum Jarzynski relation $[10,11]$.

In an open system driven by a time-dependent force, the second law states that dissipation or entropy production is the sum of the change in the system von Neumann entropy, plus the heat entering the reservoir divided by its initial temperature which represents the change in entropy in the reservoir if it were ideal (i.e., always at equilibrium). This is shown in Refs. [12-14] and in the weak coupling limit (where the reservoir is ideal) in Refs. [15-17]. A reversible transformation occurs when entropy production can be neglected. There are different ways to generate such transformations. One consists in slowly driving an open system weakly coupled to an ideal reservoir so that it will remain at any time very close to equilibrium. Since heat and entropy change are identical to first order away from equilibrium, entropy production is of second order and thus negligible. No notion of QM adiabaticity enters at this level. Another way consists in detaching the system from the reservoir and only considering a driven isolated system where the unitary dynamics leaves the system von Neumann entropy invariant and where no heat is exchanged. The second law is then empty and the first law trivial (the energy change is the mechanical work done by the driving). Once again, QM adiabaticity plays no role here.

Let us now consider the framework of the quantum Jarzynski relation derived for driven isolated systems initially prepared in a canonical equilibrium (using, for instance, a weak interaction with an ideal reservoir which is removed when the driving starts acting) $[18,19]$. In this case, fluctuations in entropy production are expressed as dissipative work (i.e., the mechanical work minus the difference in equilibrium free energy difference corresponding to the final and the initial system Hamiltonian). But this dissipation actually only occurs if one reconnects the system to its reservoir after the driving ends. This means that in this framework we are actually dealing with a specific class of driven open systems where the driving and the relaxation phase occur separately. The first phase is the driven nondissipative dynamics that brings the isolated system to a nonequilibrium state at the expense of mechanical work. The second is the nondriven dissipative dynamics starting at the end of the first phase when the system is reconnected to the ideal reservoir and ending when it has reached equilibrium. The resulting dissipation is the relative entropy distance between the nonequilibrium state produced at the end of the first phase and the equilibrium state reached at the end of the second one which equals the dissipative work $[13,20,21]$.

A special situation occurs if we consider a cyclic driving. If during the first phase the dynamics is QM adiabatic, the final state of the system will coincide with its initial equilibrium state, and no dissipation will occur during reconnection. However, for a cyclic driving generating QM-diabatic transitions, this will not be the case and dissipation will ensue. Consequently, for cyclic drivings the absence of dissipation is directly linked to QM adiabaticity. For noncyclic drivings the situation is different. Whether or not the transformation is QM adiabatic, a final nonequilibrium state will generically be produced. In this case the resulting dissipation has thus again little to do with the amount of QM-diabatic transitions.

To establish an explicit expression relating dissipation to LZ transitions, we need to go beyond these frameworks and consider driven systems coupled to reservoirs made of a finite number of levels initially at equilibrium. As the driving moves the system levels, they will cross the reservoir levels and a rich dynamics will ensue. We consider the simplest case of a single system level and treat the crossing dynamics within LZ theory.

\section{MODEL}

We assume that we have control over the energy $\epsilon_{t}$ of a single Fermionic level which constitutes the system. The reservoir is made of $L$ initially equilibrated Fermionic levels with energy $\varepsilon_{i}(i=1, \ldots, L)$, thermal occupation $f_{i} \equiv$ $f\left(\varepsilon_{i}\right)=1 /\left(e^{\beta\left(\varepsilon_{i}-\mu\right)}+1\right)$, and spacing $\Delta \varepsilon_{i+}=\varepsilon_{i+1}-\varepsilon_{i}$. As usual, $\beta^{-1}=k_{B} T$ and $T$ and $\mu$ are respectively the reservoir temperature and chemical potential. As the system level is raised, consecutive avoided crossings between the system level and the reservoir levels will occur (see Fig. 1). The raising 


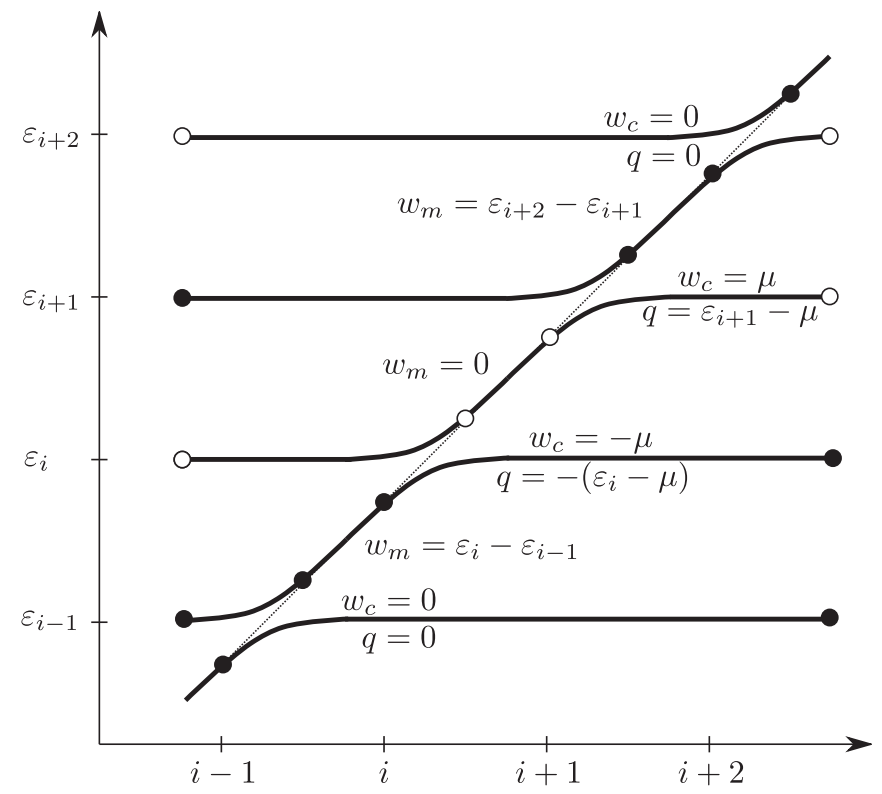

FIG. 1. Illustration of a possible trajectory when the system level $\epsilon_{t}$ is driven at constant speed $\dot{\epsilon}$ across equally spaced reservoir levels $\varepsilon_{i}$. Open (solid) circles denote empty (occupied) levels.

speed at the crossing $i$ is denoted $\dot{\epsilon}_{i}$ and is assumed to remain constant until the next crossing $i+1$ (i.e., the raising speed varies slowly between adjacent crossings). The time to go from crossing $i$ to $i+1$ is thus given by $\Delta t_{i+}=\Delta \varepsilon_{i+} / \dot{\epsilon}_{i}$ and the time at which the crossing with level $i$ occurs is $t_{i}=\sum_{j=1}^{i-1} \Delta t_{j+}$ for $i>1$ and $t_{1}=0$. The gap between the two levels at an avoided crossing $i$ is denoted $\delta_{i}$ and characterizes the system-reservoir interaction strengths. It is always assumed smaller than the spacing between the reservoir levels $\Delta \varepsilon_{i+}>\delta_{i}$, so that the system-reservoir dynamics can be treated sequentially (i.e., one reservoir level at a time) and within LZ theory [22]. The probability of a QM-diabatic (resp. QM-adiabatic) transition at the crossing $i$ is given by $R_{i}=\exp \left\{-\pi \delta_{i}^{2} /\left(2 \hbar \dot{\epsilon}_{i}\right)\right\}$ (resp. $1-R_{i}$ ). This probability has been shown to be accurate for times after the crossing longer than $t_{i}^{\mathrm{lz}}=\sqrt{\hbar / \dot{\epsilon}_{i}} \max \left[1, \sqrt{\delta_{i}^{2} /\left(\hbar \dot{\epsilon}_{i}\right)}\right][23,24]$. This means that we demand that $\Delta t_{i+}>t_{i}^{\mathrm{lz}}$, which, together with $\Delta \varepsilon_{i+}>\delta_{i}$, implies overall that our treatment requires $\Delta \varepsilon_{i+}>\sqrt{\hbar \dot{\epsilon}_{i}}, \delta_{i}$.

\section{DYNAMICS}

If $p_{i}$ is the occupation of the system level just before the avoided crossing with $\varepsilon_{i}$, the occupation of the system level a time $t_{i}^{\mathrm{tz}}$ after the avoided crossing is given by $p_{i}^{\prime}=$ $R_{i} p_{i}+\left(1-R_{i}\right) f\left(\varepsilon_{i}\right)$. As the system energy is raised until just before the next crossing at energy $\varepsilon_{i+1}$, the probability does not change and thus $p_{i+1}=p_{i}^{\prime}$. The evolution of the system occupation can therefore be rewritten as a Markov chain with transition probabilities at the crossing $i, M_{i}^{-}=$ $\left(1-R_{i}\right)\left(1-f_{i}\right)$ to empty the system level by filling the reservoir one, and $M_{i}^{+}=\left(1-R_{i}\right) f_{i}$ to fill the system level and empty the reservoir one:

$$
p_{i+1}=\left(1-M_{i}^{-}\right) p_{i}+M_{i}^{+}\left(1-p_{i}\right)
$$

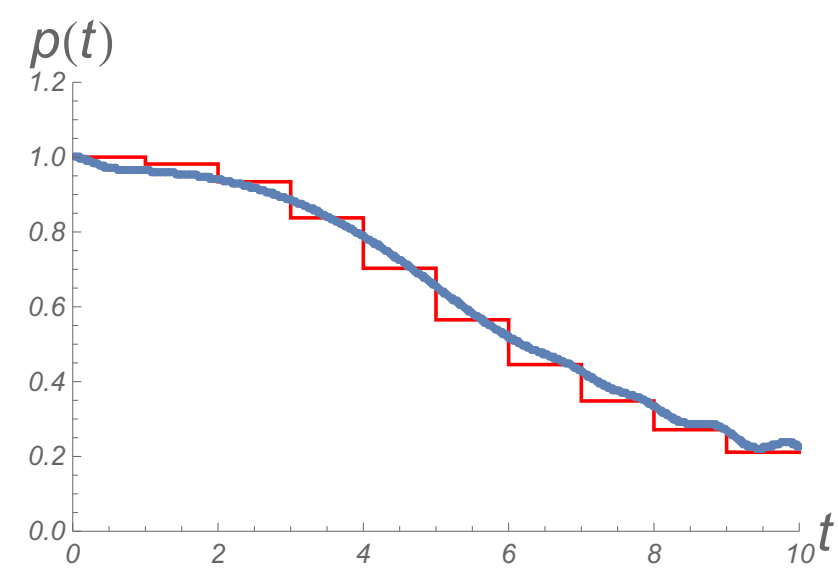

FIG. 2. System level occupation $p$ for model (2) with a protocol $\epsilon_{t}=\dot{\epsilon} t=t$. The system level crosses the $L=9$ reservoir levels with energy $\varepsilon_{i}=i \in\{1, \ldots, 9\}$ over a time $t=10$ and $\gamma=0.2, \mu=3$, $\beta=1.2$. The staircase red curve is obtained by solving the stochastic model (1) and the continuous blue curve by solving the numerically exact quantum system for $p=\left\langle c^{\dagger} c\right\rangle_{t}$ with an initial factorized state where the reservoir is in the grand-canonical state and the system level is occupied with probability 1 .

We proceed with two important remarks. First, the state of the reservoir changes as the system level sequentially crosses its levels. Second, our stochastic model neglects the coherences generated by the quantum dynamics [22]. However, our scheme is expected to hold as long as a given reservoir level is not crossed twice. In this way, its nonequilibrium state and its coherences resulting from the first interaction will not influence the system dynamics anymore. This is confirmed by Fig. 2, where we compare the system occupation predicted by our stochastic dynamics (1) with that predicted using a numerically exact system-reservoir quantum mechanical calculation, which is performed as follows. The Hamiltonian used is

$$
H(t)=\epsilon_{t} c^{\dagger} c+\sum_{i=1}^{L} \varepsilon_{i} c_{i}^{\dagger} c_{i}+\gamma \sum_{i=1}^{L}\left(c^{\dagger} c_{i}+c_{i}^{\dagger} c\right)
$$

where $\epsilon_{t}=t \dot{\epsilon}$ is the site energy for the dot, $c^{\dagger}$ and $c$ are its creation and destruction operators, and $\dot{\epsilon}$ is a constant. The system level and the $\varepsilon_{i}$ level (with creation and destruction operators $c_{i}^{\dagger}$ and $c_{i}$ ) are coupled with a strength $\gamma$ such that the gaps between the levels are given by $\delta_{i}=2 \gamma$. Since this is a noninteracting many-body system, all its properties can be obtained from single-body quantities. We thus numerically solved its exact dynamics by mapping the time-dependent single-body Schrödinger equation into a system of ordinary differential equations, using a Runge-Kutta method (RK4).

We consider two important limiting regimes of the dynamics (1). The $Q M$-adiabatic regime occurs for slow driving rates $\Delta \varepsilon>\delta \gg \sqrt{\hbar \dot{\epsilon}}$ when no LZ transition occurs because $R_{i} \rightarrow 0$ and as a result $M_{i}^{+}=f_{i}$ and $M_{i}^{-}=1-f_{i}$. In this regime, the system instantaneously thermalizes by exchanging its probability with the reservoirs. If before the crossing the system is occupied with probability $p_{i}$ and the reservoir level with probability $f_{i}$, then after the crossing the system 
occupation becomes $p_{i+1}=f_{i}$ and the reservoir one $p_{i}$. In turn, the $Q M$-diabatic regime occurs for fast driving rates in terms of LZ theory, $\Delta \varepsilon>\sqrt{\hbar \dot{\epsilon}} \gg \delta$. In this case a LZ transition always occurs at the crossing because $R_{i} \rightarrow 1$ and as a result $M_{i}^{+}=M_{i}^{-}=0$. The system and the reservoir thus remain unaffected by the crossings, $p_{i+1}=p_{i}$.

\section{THERMODYNAMICS}

The system average particle number, internal energy, and Shannon entropy just before the crossing with the reservoir level $i$ are given, respectively, by

$$
\begin{aligned}
N_{i} & =p_{i}, \quad E_{i}=\varepsilon_{i} p_{i}, \\
S_{i} & =-k_{B} p_{i} \ln p_{i}-k_{B}\left(1-p_{i}\right) \ln \left(1-p_{i}\right) .
\end{aligned}
$$

Across the avoided crossing $i$, the system occupation may change and induce as a result a change in particle number, entropy, as well as energy in the form of heat. In between the crossing with $i$ and $i+1$, the occupation remains unchanged and as a result the particle number and the entropy do not change. However, if the level is filled, the energy will change under the form of mechanical work (due to changes in the energy level) by an amount $\Delta \varepsilon_{i+}$. The average work, $W_{i+}=$ $W_{i+}^{\mathrm{m}}+W_{i+}^{\mathrm{c}}$, done on the system in going from $i$ to $i+1$ (denoted $i+$ in short) thus consists of the mechanical work $W_{i+}^{\mathrm{m}}=\left(\varepsilon_{i+1}-\varepsilon_{i}\right) p_{i+1}$ generated by the driving and the chemical work $W_{i+}^{\mathrm{c}}=\mu\left(p_{i+1}-p_{i}\right)$ needed to transfer particles from the reservoir to the system. The corresponding average heat entering the system is $Q_{i+}=\left(\varepsilon_{i}-\mu\right)\left(p_{i+1}-p_{i}\right)$. In accordance with the first and second laws of thermodynamics, the energy and entropy change can be written as

$$
\begin{gathered}
\Delta E_{i+}=E_{i+1}-E_{i}=W_{i+}+Q_{i+}, \\
\Delta S_{i+}=S_{i+1}-S_{i}=\Sigma_{i+}+Q_{i+} / T .
\end{gathered}
$$

Using the local detailed balance property of LZ rates, $M_{i}^{+} / M_{i}^{-}=e^{-\beta\left(\varepsilon_{i}-\mu\right)}$, the entropy production can be shown to be nonnegative and reads

$$
\begin{aligned}
\Sigma_{i+}= & k_{B} M_{i}^{+}\left(1-p_{i}\right) \ln \frac{M_{i}^{+}\left(1-p_{i}\right)}{M_{i}^{-} p_{i}} \\
& +k_{B} M_{i}^{-} p_{i} \ln \frac{M_{i}^{-} p_{i}}{M_{i}^{+}\left(1-p_{i}\right)}-k_{B} D\left(p_{i+1} \mid p_{i}\right) \geqslant 0,
\end{aligned}
$$

where $\quad D\left(p \mid p^{\prime}\right)=p \ln \left[p / p^{\prime}\right]+(1-p) \ln [(1-p) /(1-$ $\left.\left.p^{\prime}\right)\right] \geqslant 0$ denotes the relative entropy. The detailed calculations for a general stochastic thermodynamics in discrete time are given in the Appendix. Combining the first and second laws, the entropy production can also be rewritten as $T \Sigma_{i+}=W_{i+}-\Delta \Omega_{i+}$, where $\Delta \Omega_{i+}=\Omega_{i+1}-\Omega_{i}$ is the change in nonequilibrium grand potential $\Omega_{i}=E_{i}-\mu N_{i}-T S_{i}$. Introducing the dissipated mechanical work $W_{i+}^{\text {diss }}=W_{i+}^{\mathrm{m}}-\Delta \Omega_{i+}^{\mathrm{eq}}$ with the equilibrium grand potential $\Omega_{i}^{\mathrm{eq}}=k_{B} T \ln \left(1-f_{i}\right)$, and realizing that $\Omega_{i}-\Omega_{i}^{\mathrm{eq}}=k_{B} T D\left(p_{i} \mid f_{i}\right)$, we find that entropy production can now be expressed as

$$
\Sigma_{i+}=\frac{W_{i+}^{\text {diss }}}{T}-k_{B} D\left(p_{i+1} \mid f_{i+1}\right)+k_{B} D\left(p_{i} \mid f_{i}\right) \geqslant 0 .
$$

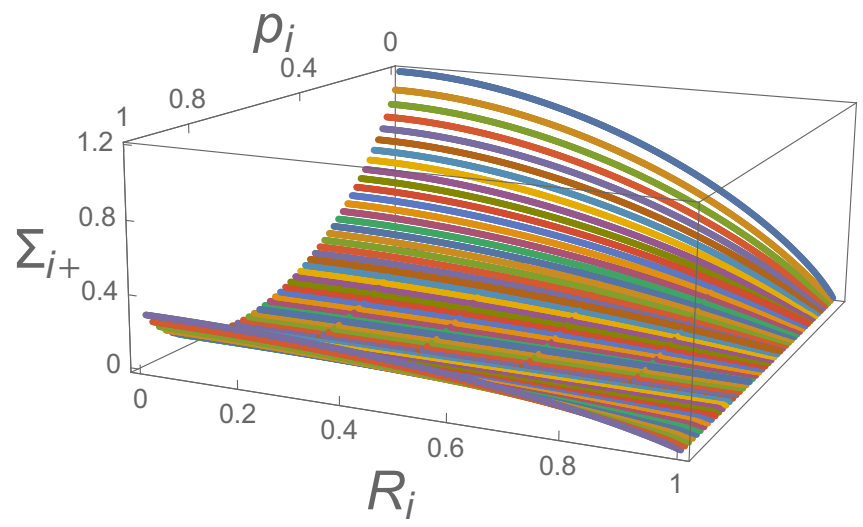

FIG. 3. Entropy production (6) as a function of the rate of QMdiabatic transitions $R$ and of the initial system level occupation $p_{i}$ before the crossing, for $f_{i}=0.75$.

The second (third) term on the right-hand side measures the distance from equilibrium right before crossing $i+1(i)$.

We now consider the thermodynamics of the QM-adiabatic regime. If just before the crossing the system has been prepared in an arbitrary occupation $p_{i}$, using (6) and (7), we find that the dissipation occurring at the crossing is $\Sigma_{i+}=k_{B} D\left(p_{i} \mid f_{i}\right)$ and the dissipative work done on the system to lift the level from $i$ to $i+1$ is $W_{i+}^{\text {diss }}=k_{B} T D\left(f_{i} \mid f_{i+1}\right)$. If the system is initially prepared at equilibrium, $p_{1}=f_{1}$ and the first crossing is perfectly reversible: $\Sigma_{1+}=0$. However, the instantaneous thermalization of the level implies that the subsequent crossings $(i>1)$ will be such that $p_{i}=f_{i-1}$ and $p_{i+1}=f_{i}$ and an amount $\Sigma_{i+}=k_{B} D\left(f_{i-1} \mid f_{i}\right)$ will be dissipated at every crossing. We therefore conclude that in general QM adiabaticity does not imply thermodynamic reversibility. This, however, becomes true in the limit $\Delta \varepsilon, \delta, \dot{\epsilon} \rightarrow 0$ where the inequality $\Delta \varepsilon>\delta \gg \sqrt{\hbar \dot{\epsilon}}$ is maintained. Indeed, in this case $\Sigma_{i+}$ as well as $W_{i+}^{\text {diss }}$ both tend to zero proportionally to order $\Delta \varepsilon^{2}$ while heat and entropy change become equal, to order $\Delta \varepsilon$. We now turn to the thermodynamics of the QM-diabatic regime. In this case, the level rises without changes in its initial occupation probability $p_{1}$. As a result no dissipation occurs $(\Sigma=0)$, and no entropy or heat is produced. Only work is done to lift the energy of the level and $W^{\text {diss }}=k_{B} T \sum_{i}\left(D\left(p_{1} \mid f_{i+1}\right)-D\left(p_{1} \mid f_{i}\right)\right)$.

The dissipation (6) or (7) across a transition is represented in Fig. 3 as a function of the rate of QM-diabatic transitions, $R$, and of the initial system level occupation $p_{i}$ before the crossing and for $f_{i}=0.75$. As announced, the dissipation vanishes when $R \rightarrow 1$ (QM-diabatic limit) independently of $p_{i}$ or when $R \rightarrow 0$ (QM-adiabatic limit) if $p_{i}=f_{i}$.

\section{MECHANICAL WORK FLUCTUATIONS}

We can use our stochastic thermodynamic description of the system to study mechanical work fluctuations. Thanks to the local detailed balance property of the rates, the work fluctuation theorem can be derived following a procedure almost identical to that detailed in Refs. [25-27] and reads $\ln P\left(w^{\mathrm{m}}\right) / \tilde{P}\left(-w^{\mathrm{m}}\right)=\beta\left(w^{\mathrm{m}}-\Delta \Omega^{\mathrm{eq}}\right) . \quad P\left(w^{\mathrm{m}}\right)$ denotes the probability that the external force performs a mechanical 


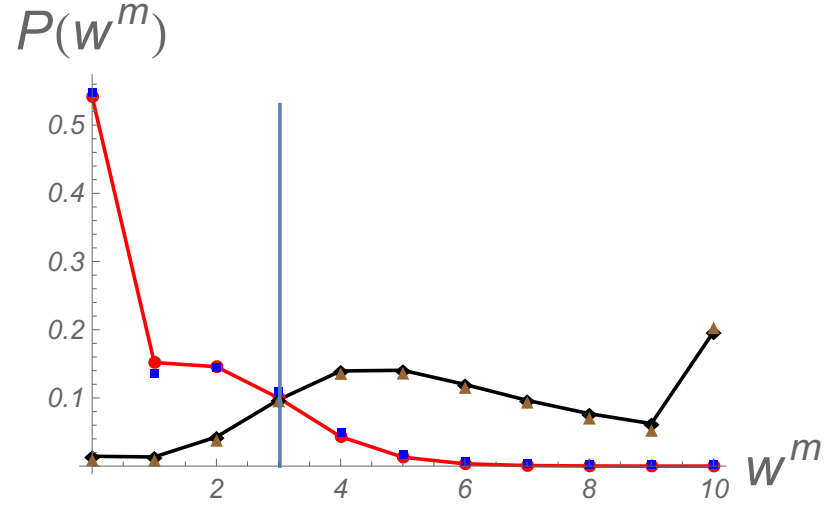

FIG. 4. Forward $P\left(w^{\mathrm{m}}\right)$ [backward $\tilde{P}\left(-w^{\mathrm{m}}\right)$ ] work distribution calculated using LZ theory, in black (red), and using the full quantum dynamics, in brown (blue). The vertical line marks the value of $\Delta \Omega^{\mathrm{eq}}$. Same parameters as Fig. 2.

work $w^{\mathrm{m}}$ when driving the system (initially at equilibrium) according to a given forward protocol. $\tilde{P}\left(w^{\mathrm{m}}\right)$ denotes the same probability when the driving protocol is time reversed and the system is initially at equilibrium with respect to the final value of the forward driving protocol. In Fig. 4, these two distributions obtained using our stochastic model are shown to be in excellent agreement with those obtained using the numerically exact quantum dynamics in the total system with Hamiltonian (2). In this latter case, the mechanical work is obtained from the energy changes resulting from a two point projective measurement of the total system energy at the beginning and at the end of the process $[10,11,18,19]$.

\section{CONTINUOUS TIME LIMIT}

We now consider that we operate close to the QM-diabatic regime where $\Delta \varepsilon>\sqrt{\hbar \dot{\epsilon}}>\delta$ and we assume that the coupling $\delta_{i}$, the driving $\dot{\epsilon}_{i}$, and the reservoir density of states $d=1 / \Delta \varepsilon$ vary smoothly with $i$. In this regime, the rate of QM-diabatic transition can be expanded as $R \approx 1-\frac{\delta^{2}}{\hbar \dot{\epsilon}} \frac{\pi}{2}$. Introducing the reservoir density of states $d_{i}=1 / \Delta \varepsilon_{i}$ and remembering that $\Delta t_{i+}=1 /\left(\dot{\epsilon}_{i} d_{i}\right)$, the dynamics $\left(p_{i+1}-p_{i}\right) / \Delta t_{i+}=\left(f_{i}-p_{i}\right)\left(1-R_{i}\right) / \Delta t_{i+}$ can be treated as a continuous time master equation $d_{t} p=w^{+}(1-p)-w^{-} p$ with Fermi golden rule rates

$$
\begin{gathered}
w^{+}=\frac{\pi \delta^{2}\left(\epsilon_{t}\right) d\left(\epsilon_{t}\right)}{2 \hbar} f\left(\epsilon_{t}\right), \\
w^{-}=\frac{\pi \delta^{2}\left(\epsilon_{t}\right) d\left(\epsilon_{t}\right)}{2 \hbar}\left[1-f\left(\epsilon_{t}\right)\right] .
\end{gathered}
$$

These rates satisfy local detailed balance and thus a consistent stochastic thermodynamics ensues [28-30]. Note that their explicit dependence on the driving speed $\dot{\epsilon}_{i}$ has disappeared. The restriction to be close to the QM-diabatic regime, however, puts the limit of reversible transformations outside the realm of validity of this description. It is interesting to note that exactly the same dynamics can be derived by assuming that the system is weakly coupled to a continuous reservoir in the BornMarkov secular approximation [31]. However, in this case the above restriction does not hold and the limit of reversible transformations is reachable. This may indicate that the restriction $\Delta \varepsilon>\delta$ could be loosened as also suggested by the results of Ref. [32].

\section{CONCLUSIONS}

We established a stochastic thermodynamics for a single system level interacting with a finite set of initially thermalized reservoir levels. This enabled us to derive an explicit expression for dissipation in terms of rates of QM-diabatic transitions. Thermodynamically reversible transformations require QM adiabaticity but also a vanishing reservoir level spacing as well as a vanishing system-reservoir interacting strength. Our treatment can be immediately generalized to multiple system levels as long as the system energies do not cross. This latter case with crossings would require some more care. We emphasize that our study is very different from other studies which considered the crossing dynamics between system levels in contact with continuous reservoirs [5,33-36].

\section{ACKNOWLEDGMENTS}

This work was made possible by the COST Action MP1209. F.B. acknowledges support from Fondecyt Grant No. 1151390. M.E. is supported by the National Research Fund, Luxembourg (Project No. FNR/A11/02).

\section{APPENDIX: STOCHASTIC THERMODYNAMICS IN DISCRETE TIME}

The stochastic process considered in the paper is a special case of a Markov chain in discrete time satisfying local detailed balance. We now derive stochastic thermodynamics for this general case. We consider discrete times $i=0,1,2, \ldots$ and a finite state space $m=0,1,2, \ldots, M$. The probability to find the system in state $m$ at time $i$ is denoted $p_{m}(i)$ and evolves according to the Markov chain

$$
p_{m}(i+1)=\sum_{m^{\prime}} M_{m m^{\prime}}(i) p_{m^{\prime}}(i),
$$

where the transition matrix satisfies $\sum_{m} M_{m m^{\prime}}(i)=1$. We further decompose the latter into contributions from different reservoirs $v=1,2, \ldots, R: M_{m m^{\prime}}(i)=\sum_{v} M_{m m^{\prime}}^{(v)}(i)$. We also introduce the time-dependent energy $e_{m}(i)$ and the number of particles, $n_{m}$, of state $m$ and assume that the transition matrix satisfies local detailed balance

$$
\ln \frac{M_{m m^{\prime}}^{(v)}(i)}{M_{m^{\prime} m}^{(v)}(i)}=-\frac{\left(e_{m}(i)-e_{m^{\prime}}(i)\right)-\mu_{v}(i)\left(n_{m}-n_{m^{\prime}}\right)}{T_{v}(i)},
$$

where $T_{\nu}$ and $\mu_{v}$ are the temperature and chemical potential of reservoir $v$ and $k_{B}=1$. The model considered in our paper has two states: $m=0$ when the level is empty and $m=1$ when it is occupied with probability $p_{0}=1-p$ (respectively $p_{1}=p$ ). Furthermore, $n_{0}=0, e_{0}=0, n_{1}=1$, and $e_{1}(i)=\epsilon(i)$.

The average system energy and number of particles are given by

$$
\begin{gathered}
E(i)=\sum_{m} e_{m}(i) p_{m}(i) \\
N(i)=\sum_{m} n_{m} p_{m}(i)
\end{gathered}
$$


The energy and particle current entering the system from reservoir $v$ are

$$
\begin{gathered}
I_{E}^{v}(i)=\sum_{m, m^{\prime}}\left(e_{m}(i)-e_{m^{\prime}}(i)\right) M_{m m^{\prime}}^{v}(i) p_{m^{\prime}}(i), \\
I_{M}^{v}(i)=\sum_{m, m^{\prime}}\left(n_{m}-n_{m^{\prime}}\right) M_{m m^{\prime}}^{v}(i) p_{m^{\prime}}(i),
\end{gathered}
$$

and the average heat entering the system from reservoir $v$ is

$$
Q^{v}(i)=I_{E}^{v}(i)-\mu_{\nu} I_{M}^{v}(i) .
$$

The average work done on the system is made of mechanical and chemical work:

$$
\begin{aligned}
W(i) & =W_{\text {mech }}(i)+W_{\text {chem }}(i), \\
W_{\text {mech }}(i) & =\sum_{m}\left(e_{m}(i+1)-e_{m}(i)\right) p_{m}(i+1), \\
W_{\text {chem }}(i) & =\sum_{\nu} \mu_{\nu} I_{M}^{v}(i) .
\end{aligned}
$$

The first law of thermodynamics ensues and is given by

$$
\Delta E(i)=E(i+1)-E(i)=W(i)+\sum_{v} Q^{v}(i) .
$$

We now define the Shannon entropy of the system:

$$
S(i)=-\sum_{m} p_{m}(i) \ln p_{m}(i) .
$$

The second law of thermodynamics reads

$$
\Delta S(i) \equiv S(i+1)-S(i)=\sum_{v} \frac{Q^{(v)}(i)}{T_{v}}+\Sigma(i),
$$

where the entropy flow is given by

$$
\sum_{\nu} \frac{Q^{(v)}(i)}{T_{\nu}}=-\sum_{\nu, m, m^{\prime}} M_{m m^{\prime}}^{(v)}(i) p_{m^{\prime}}(i) \ln \frac{M_{m m^{\prime}}^{(v)}(i)}{M_{m^{\prime} m}^{(\nu)}(i)}
$$

and the entropy production is defined as

$$
\Sigma(i)=\sum_{\nu, m, m^{\prime}} M_{m m^{\prime}}^{(v)}(i) p_{m^{\prime}}(i) \ln \frac{M_{m m^{\prime}}^{(v)}(i) p_{m^{\prime}}(i)}{M_{m^{\prime} m}^{(v)}(i) p_{m}(i+1)} .
$$

This quantity is non-negative as can be shown using the inequality $-\ln X \geqslant X-1$. Indeed,

$$
\begin{aligned}
\Sigma(i) & \geqslant \sum_{\nu, m, m^{\prime}} M_{m m^{\prime}}^{(v)}(i) p_{m^{\prime}}(i)\left(\frac{M_{m^{\prime} m}^{(v)}(i) p_{m}(i+1)}{M_{m m^{\prime}}^{(v)}(i) p_{m^{\prime}}(i)}-1\right) \\
& =\sum_{\nu, m, m^{\prime}}\left(M_{m^{\prime} m}^{(v)}(i) p_{m}(i+1)-M_{m m^{\prime}}^{(v)}(i) p_{m^{\prime}}(i)\right)=0 .
\end{aligned}
$$

It is zero when detailed balance is satisfied, i.e., when

$$
M_{m m^{\prime}}^{(\nu)}(i) p_{m^{\prime}}(i)=M_{m^{\prime} m}^{(\nu)}(i) p_{m}(i+1) .
$$

The entropy production can also be rewritten as

$$
\begin{aligned}
\Sigma(i)= & \sum_{\nu, m, m^{\prime}} M_{m m^{\prime}}^{(v)}(i) p_{m^{\prime}}(i) \ln \frac{M_{m m^{\prime}}^{(v)}(i) p_{m^{\prime}}(i)}{M_{m^{\prime} m}^{(v)}(i) p_{m}(i)} \\
& -\sum_{m} p_{m}(i+1) \ln \frac{p_{m}(i+1)}{p_{m}(i)} .
\end{aligned}
$$

The first term is the non-negative term that survives in the continuous time limit (see, e.g., Ref. [37]) while the second one is negative and vanishes. A similar expression was also found in Refs. [38,39].
[1] L. Landau, Phys. Z. Sowjetunion 2, 46 (1932).

[2] C. Zener, Proc. R. Soc. London A 137, 696 (1932).

[3] E. Majorana, Nuovo Cimento 9, 43 (1932).

[4] E. Stueckelberg, Helv. Phys. Acta 5, 369 (1932).

[5] A. Dodin, S. Garmon, L. Simine, and D. Segal, J. Chem. Phys. 140, 124709 (2014).

[6] M. Wilkinson, J. Phys. A: Math. Gen. 21, 4021 (1988).

[7] M. Wilkinson, Physica A (Amsterdam, Neth.) 157, 58 (1989).

[8] A. Polkovnikov, Ann. Phys. 326, 486 (2011).

[9] A. Polkovnikov, K. Sengupta, A. Silva, and M. Vengalattore, Rev. Mod. Phys. 83, 863 (2011).

[10] M. Esposito, U. Harbola, and S. Mukamel, Rev. Mod. Phys. 81, 1665 (2009).

[11] M. Campisi, P. Hänggi, and P. Talkner, Rev. Mod. Phys. 83, 771 (2011).

[12] M. Esposito, K. Lindenberg, and C. Van den Broeck, New J. Phys. 12, 013013 (2010).

[13] M. Esposito and C. Van den Broeck, Europhys. Lett. 95, 40004 (2011).

[14] D. Reeb and M. M. Wolf, New J. Phys. 16, 103011 (2014).

[15] H. Spohn and J. L. Lebowitz, in Irreversible Thermodynamics for Quantum Systems Weakly Coupled to Thermal Reservoirs (John Wiley \& Sons, New York, 2007), pp. 109-142.

[16] R. Alicki, J. Phys. A 12, L103 (1979).
[17] R. Kosloff, Entropy 15, 2100 (2013).

[18] H. Tasaki, arXiv:cond-mat/0009244.

[19] J. Kurchan, arXiv:cond-mat/0007360.

[20] R. Kawai, J. M. R. Parrondo, and C. Van den Broeck, Phys. Rev. Lett. 98, 080602 (2007).

[21] S. Vaikuntanathan and C. Jarzynski, Europhys. Lett. 87, 60005 (2009).

[22] S. N. Shevchenko, S. Ashhab, and F. Nori, Phys. Rep. 492, 1 (2010).

[23] K. Mullen, E. Ben-Jacob, Y. Gefen, and Z. Schuss, Phys. Rev. Lett. 62, 2543 (1989).

[24] N. V. Vitanov, Phys. Rev. A 59, 988 (1999).

[25] G. E. Crooks, Phys. Rev. E 61, 2361 (2000).

[26] G. E. Crooks, J. Stat. Phys. 90, 1481 (1998).

[27] G. Bulnes Cuetara, M. Esposito, and A. Imparato, Phys. Rev. E 89, 052119 (2014).

[28] U. Seifert, Rep. Prog. Phys. 75, 126001 (2012).

[29] M. Esposito, Phys. Rev. E 85, 041125 (2012).

[30] C. Van den Broeck and M. Esposito, Physica A 418, 6 (2015).

[31] H.-P. Breuer and F. Petruccione, The Theory of Open Quantum Systems (Oxford University Press, Oxford, U.K., 2002).

[32] Y. Kayanuma and S. Fukuchi, J. Phys. B: At. Mol. Phys. 18, 4089 (1985). 
[33] P. Ao and J. Rammer, Phys. Rev. B 43, 5397 (1991).

[34] E. Shimshoni and Y. Gefen, Ann. Phys. 210, 16 (1991).

[35] W. K. Lai, K.-A. Suominen, B. M. Garraway, and S. Stenholm, Phys. Rev. A 47, 4779 (1993).
[36] M. Yamaguchi, T. Yuge, and T. Ogawa, arXiv:1604.01082.

[37] P. Gaspard, J. Stat. Phys 117, 599 (2004).

[38] B. Altaner and J. Vollmer, arXiv:1212.4728.

[39] B. Altaner, Ph.D. thesis, University of Göttingen, 2014. 